\title{
Should CYP2D6 be genotyped when treating with tamoxifen?
}

\author{
"The highly polymorphic CYP2D6 gene is the principal enzyme \\ of tamoxifen biotransformation to its major active metabolite, \\ endoxifen."
}

First draft submitted: 5 October 2016; Accepted for publication: 5 October 2016; Published online: 24 November 2016

Keywords: CYP2D6 • drug-drug interaction • pharmacogenetics • tamoxifen

In patients with estrogen receptor positive $\left(\mathrm{ER}^{+}\right)$breast cancer, the response to tamoxifen can differ among individuals [1]. The highly polymorphic CYP2D6 gene is the principal enzyme of tamoxifen biotransformation to its major active metabolite, endoxifen [1]. Over the past few years, research has placed great emphasis on the variability of clinical efficacy of tamoxifen, suggesting a correlation between drug metabolism and CYP2D 6 genotype of patients [2]. Many studies aimed at identifying the role of different CYP2D6 variants found significant association between genotype, phenotype and clinical outcome of patients. Goetz et al. showed that patients carrying the CYP2D6 * $4 / * 4$ genotype have a higher risk of disease relapse and a lower incidence of adverse drug reactions, due to the lower metabolic activation of tamoxifen to endoxifen [3]. Schroth et al. analysed 206 patients receiving adjuvant tamoxifen monotherapy and 280 patients not receiving tamoxifen therapy (71 months median follow-up), and concluded that genotyping for CYP2D6 *4, *5, *10 and ${ }^{*} 41$ variants help identify patients who will have little benefit from adjuvant tamoxifen therapy [4]. However, conflicting data are reported on how CYP2D6 variants could be important determinants of tamoxifen activity and there are few guidelines linking the results of pharmacogenetic tests to specific therapeutic recommendations. Several clinical trials are reported in literature, with results ranging from a longer disease-free survival interval [5] to a shorter recurrencefree survival time [6] for patients carrying the CYP2D6 poor metabolizer phenotype. Many factors may have influenced the outcome of studies on CYP2D6 and its association with tamoxifen efficacy, i.e., tamoxifen combination with inhibitors of CYP2D6 enzyme and treatment compliance. The ATAC [7] and the BIG 1-98 [8] trials examined the associations between CYP2D6 genotype and tamoxifen treatment in 1203 and 4392 patients, respectively. None of these studies observed a correlation between CYP2D 6 genotype and clinical outcome with tamoxifen. Nevertheless, many aspects of the ATAC and BIG 1-98 have to be considered: the most important argument is the use of formalin-fixed paraffin-embedded tumor tissues for CYP2D 6 genotyping, instead of germ-line DNA. The use of formalin-fixed paraffin-embedded samples may lead to an important deviation from the Hardy-Weinberg equilibrium (expected vs observed genotype frequencies) for two major reasons: generation of nonspecific products during the polymerase chain reaction [9] and the presence of loss of heterozygosity at the CYP2D 6 locus, resulting in genotyping errors [10]. This issue was confirmed in both the ATAC and BIG 1-98 studies, where the Hardy-Weinberg equilibrium was not respected, with a substantial

\begin{abstract}
Marzia Del Re
Clinical Pharmacology

\& Pharmacogenetics Unit, Department of Clinical \& Experimental Medicine, University of Pisa, 55 Via Roma, 56126 Pisa, Italy
\end{abstract}

Eleonora Rofi

Clinical Pharmacology \& Pharmacogenetics Unit, Department of Clinical \& Experimental Medicine, University of Pisa, 55 Via Roma, 56126 Pisa, Italy

Valentina Citi

Clinical Pharmacology

\& Pharmacogenetics Unit, Department of Clinical \& Experimental Medicine, University of Pisa, 55 Via Roma, 56126 Pisa, Italy

\section{Leonardo Fidilio}

Clinical Pharmacology \& Pharmacogenetics Unit, Department of Clinical \& Experimental Medicine, University of Pisa, 55 Via Roma, 56126 Pisa, Italy

Romano Danesi

Author for correspondence:

Clinical Pharmacology

\& Pharmacogenetics Unit, Department of Clinical \& Experimental Medicine, University of Pisa, 55 Via Roma, 56126 Pisa, Italy

Tel.: + 0039050992632

Fax: 00390502218758

romano.danesi@unipi.it 
deviation from normal values: $\mathrm{p}=0.000021$ for the ATAC study and $\mathrm{p}=2.5 \times 10^{-92}$ for BIG 1-98 trial $[5,7]$. Owing to these controversial results, available recommendations do not suggest the use of CYP2D 6 genetic testing to select the best endocrine therapy regimen for patients. In 2006, the US FDA updated tamoxifen label including the information on the increased risk of breast cancer recurrence in poor metabolizer patients, but the subcommittee did not reach a consensus to recommend the CYP2D6 genotyping [11], and the same conclusion was reached by the National Comprehensive Cancer Network [12]. Additionally, the 2010 guidelines of the American Society of Clinical Oncology [13] and the St. Gallen's expert consensus [14] judged the available data on CYP2D6 pharmacogenetics insufficient to recommend testing as a tool to determine an adjuvant endocrine strategy. In contrast, on the basis of several studies reporting phenotype and/or genotype data from patients or healthy volunteers [3,15-17] and considering the increased risk for relapse as the main outcome measure, the Dutch Pharmacogenetics Working Group suggested the use of aromatase inhibitors for postmenopausal women with CYP2D6 poor metabolizer phenotype. Moreover, for intermediate metabolizers, the guidelines suggested the use of aromatase inhibitors or the avoidance of concomitant CYP2D6 inhibitors, if tamoxifen is the first choice for the specific patient [18]. Indeed, clinicians should be advised that also drug-drug interactions may influence endoxifen plasma concentrations, including co-administration of other drugs, such as selective serotonin reuptake

\section{References}

1 De Souza JA, Olopade OI. CYP2D6 genotyping and tamoxifen: an unfinished story in the quest for personalized medicine. Semin. Oncol. 38(2), 263-273 (2011).

2 Kaplan M, Mahon SM. Tamoxifen benefits and CYP2D6 testing in women with hormone receptor-positive breast cancer. Clin. J. Oncol. Nurs. 17(2), 174-179 (2013).

3 Goetz MP, Rae JM, Suman VJ et al. Pharmacogenetics of tamoxifen biotransformation is associated with clinical outcomes of efficacy and hot flashes. J. Clin. Oncol. 23(36), 9312-9318 (2005).

4 Schroth W, Antoniadou L, Fritz P et al. Breast cancer treatment outcome with adjuvant tamoxifen relative to patient CYP2D6 and CYP2C19 genotypes. J. Clin. Oncol. 25(33), 5187-5193 (2007).

5 Regan MM, Leyland-Jones B, Bouzyk M et al. CYP2D6 genotype and tamoxifen response in postmenopausal women with endocrine-responsive breast cancer: the Breast International Group 1-98 trial. J. Natl Cancer Inst. 104(6), 441-451 (2012).

6 Dezentje VO, Van Schaik RH, Vletter-Bogaartz JM et al. CYP2D6 genotype in relation to tamoxifen efficacy in a Dutch cohort of the Tamoxifen Exemestane Adjuvant inhibitors, which interfere with the enzymatic conversion of tamoxifen to endoxifen by inhibiting CYP2D6 enzyme [17,19]. Interestingly, the consensus of the National Comprehensive Cancer Network Breast Cancer Risk Reduction Panel is that "....alternative medications that have minimal or no impact on plasma levels of endoxifen should be substituted when possible" even though CYP2D6 genotyping is not recommended as further validation of this biomarker within the clinical setting is required [20].

To conclude, even though the available recommendations are against the CYP2D 6 screening, the negative results of clinical trials may have been biased by issues in data collection, analysis and interpretation. Hopefully, these recommendations will be revised with the contribution of experts in clinical pharmacogenetics and drug metabolism, in order not to underestimate the negative impact of CYP2D6 inhibitors that may be administered during tamoxifen therapy and potentially decrease the concentrations of the active metabolite endoxifen.

\section{Financial \& competing interests disclosure}

The authors have no relevant affiliations or financial involvement with any organization or entity with a financial interest in or financial conflict with the subject matter or materials discussed in the manuscript. This includes employment, consultancies, honoraria, stock ownership or options, expert testimony, grants or patents received or pending, or royalties.

No writing assistance was utilized in the production of this manuscript.

Multinational (TEAM) trial. Breast Cancer Res. Treat 140(2), 363-373 (2013).

7 Rae JM, Drury S, Hayes DF et al. CYP2D6 and UGT2B7 genotype and risk of recurrence in tamoxifen-treated breast cancer patients. J. Natl Cancer Inst. 104(6), 452-460 (2012).

8 Regan MM, Price KN, Giobbie-Hurder A et al. Interpreting Breast International Group (BIG) 1-98: a randomized, double-blind, Phase III trial comparing letrozole and tamoxifen as adjuvant endocrine therapy for postmenopausal women with hormone receptor-positive, early breast cancer. Breast Cancer Res. 13(3), 209 (2011).

9 Bell DA, Demarini DM. Excessive cycling converts PCR products to random-length higher molecular weight fragments. Nucleic Acids Res. 19(18), 5079 (1991).

10 Goetz MP, Sun JX, Suman VJ et al. Loss of heterozygosity at the CYP2D6 locus in breast cancer: implications for germline pharmacogenetic studies. J. Natl Cancer Inst. 107(2), (2015).

11 Danesi R, Del Re M, Ciccolini J et al. Prevention of fluoropyrimidine toxicity: do we still have to try our patient's luck? Ann. Oncol. doi:10.1093/annonc/mdw448 (2016) (Epub ahead of print). 
12 Fogli S, Saba A, Del Re M et al. Application of a pharmacokinetic/pharmacogenetic approach to assess the nicotine metabolic profile of smokers in the real-life setting. J. Pharm. Biomed. Anal. 131, 208-213 (2016).

13 Harris LN, Ismaila N, Mcshane LM et al. Use of biomarkers to guide decisions on adjuvant systemic therapy for women with early-stage invasive breast cancer: American Society of Clinical Oncology Clinical Practice Guideline. J. Clin. Oncol. 34(10), 1134-1150 (2016).

14 Gnant M, Harbeck N, Thomssen C. St. Gallen 2011 summary of the consensus discussion. Breast Care (Basel) 6(2), 136-141 (2011).

15

Bonanni B, Macis D, Maisonneuve P et al. Polymorphism in the $C Y P 2 D 6$ tamoxifen-metabolizing gene influences clinical effect but not hot flashes: data from the Italian tamoxifen trial. J. Clin. Oncol. 24(22), 3708-3709; author reply 3709 (2006).
16 Borges S, Desta Z, Li L et al. Quantitative effect of CYP2D6 genotype and inhibitors on tamoxifen metabolism: implication for optimization of breast cancer treatment. Clin. Pharmacol. Ther. 80(1), 61-74 (2006).

17 Jin Y, Desta Z, Stearns V et al. CYP2D6 genotype, antidepressant use, and tamoxifen metabolism during adjuvant breast cancer treatment. J. Natl Cancer Inst. 97(1), 30-39 (2005).

18 Swen JJ, Nijenhuis M, De Boer A et al. Pharmacogenetics: from bench to byte-an update of guidelines. Clin. Pharmacol. Ther. 89(5), 662-673 (2011).

19 Sideras K, Ingle JN, Ames MM et al. Coprescription of tamoxifen and medications that inhibit CYP2D6. J. Clin. Oncol. 28(16), 2768-2776 (2010).

20 Bevers TB, Armstrong DK, Arun B et al. Breast cancer risk reduction. J. Natl Compr. Canc. Netw. 8(10), 1112-1146 (2010). 